\title{
A trajetória dos estudos sobre a agenda de políticas públicas
}

\author{
Policy agenda studies: a brief history
}

\author{
Ana Cláudia Niedhardt Capella \\ Doutora em Ciências Sociais, Professora colaboradora \\ do Programa de Pós-Graduação em Ciência Política, \\ Universidade Federal de São Carlos (UFSCar), \\ Docente do Departamento de Administração Pública, \\ Universidade Estadual Paulista (UNESP), Araraquara, \\ SP, Brasil \\ acapella@terra.com.br
}

\author{
Felipe Gonçalves Brasil \\ Bacharel em Gestão de Políticas Públicas, Universidade \\ de São Paulo (USP), Escola de Artes, Ciências e \\ Humanidades (EACH), Mestre e Doutorando em Ciência \\ Política, Universidade Federal de São Carlos (UFSCar) \\ e bolsista FAPESP. Foi professor substituto na Unesp \\ Araraquara e Intern Student na North Carolina State \\ University, Raleigh, Estados Unidos \\ fbrasil.pp@gmail.com
}

Resumo: Este estudo tem como objetivo retomar o histórico dos estudos de agenda no campo da Ciência Política e das Políticas Públicas, destacando as tendências contemporâneas de pesquisa na área e os esforços de elaboração teórico-metodológicos voltados à investigação desse tema. Para tanto identificaremos quatro momentos principais: o início das investigações sobre agenda no campo da ciência política, nos anos 1970; os desenvolvimentos teóricos produzidos no campo de políticas públicas, a partir de meados dos anos 1980 e década de 1990; a expansão dos estudos de agenda, que passam a ser entendidos também como explicações sobre a dinâmica das políticas públicas, ou seja, sobre processos de mudança, nos anos 2000; e o desenvolvimento mais recente, de investigação das dinâmicas das políticas públicas em perspectiva comparada, nos anos 2010.

Palavras-chave: Agenda-setting; Agenda governamental; Equilíbrio pontuado.

\begin{abstract}
This study aims to provide an overview of the history of the agenda-setting studies developed between the fields of Political Science and Public Policy, highlighting contemporary tendencies of research as well as theoretical and methodological developments. We will point out four main stages: the initial investigations about agenda-setting in the fields of political science in the 1970s; theoretical and methodological developments produced in the public policy field, from the mid-1980s and 1990s; the expansion of agenda studies, which are also seen as explanations of the policy dynamics, that is, about the processes of policy change, in the 2000s; and, the latest stage, researches on public policies dynamics in comparative perspective, in the 2010 s.
\end{abstract}

Keywords: Agenda-setting; Policy agenda; Punctuated equilibrium. 


\section{Introdução}

$\mathrm{O}$ processo de agenda-setting tem sido objeto de questionamento e investigação pela academia, tendo sido analisado por diferentes áreas do conhecimento, como a Comunicação e a Ciência Política, com base em diferentes tradições de estudos. Enquanto os estudos na Comunicação desenvolveram-se mais voltados para a compreensão da relação entre a comunicação de massa e a opinião pública, ou seja, na relação entre a agenda midiática (media agenda-setting) e a agenda pública (public agenda-setting), na Ciência Política o foco foi deslocado para o entendimento de outras questões vinculadas ao poder político e à atenção governamental. Entendida como a policy agenda-setting, ou a agenda das políticas públicas, essa tradição de estudos desenvolve-se como um desdobramento de análises voltadas à investigação de processos de participação política e seus limites no contexto da teoria democrática, avançando para a teorização sobre a formação da agenda governamental. Autores como Cobb e Elder (1971) buscaram investigar elementos que explicassem a estruturação de uma agenda de debates dentro de uma comunidade, ou seja, os temas e problemas considerados centrais, procurando compreender como uma agenda é construída e quem participa desse processo. Dessa forma, foram conduzidos os primeiros estudos sistemáticos sobre as relações entre a agenda sistêmica (a agenda mais geral de uma comunidade) e a agenda governamental (ou agenda institucional, conjunto de temas que ocupam os tomadores de decisão) no campo da Ciência Política (COBB; ELDER, 1971, 1972).

Outra contribuição importante para a o estudo da formação da agenda foi desenvolvida por Downs (1972) que defende a existência de um ciclo de atenção responsável por influenciar o comportamento do público em relação aos problemas e, consequentemente, sobre a agenda. A percepção dos problemas, para Downs, é cíclica, iniciando-se em uma fase de (a) "pré-problema", na qual uma condição social existente ainda não chama a atenção do público; depois passando a (b) um momento de "descoberta e entusiasmo", na qual o público se torna consciente de uma questão e alarmado com suas consequências; seguindo para um momento de (c) percepção sobre as dificuldades e os custos envolvidos na resolução do problema; até que (d) o interesse do público sobre a questão começa a declinar gradualmente; e finalmente, (e) saída do problema do centro das preocupações do público, seja por meio da substituição do problema por outro ou seu deslocamento para fora do debate público, onde recebe um nível muito menor de atenção.

Os estudos sobre o processo de produção de políticas, desenvolvidos em meados dos anos 1980 e 1990 nos Estados Unidos, deram novo fôlego às investigações sobre a agenda. Esses estudos mudaram o foco das investigações da agenda sistêmica (as questões e problemas que despertam a atenção do público) para uma investigação centrada no estudo da agenda governamental (questões e problemas que ocupam os formuladores de políticas). O modelo de Múltiplos Fluxos, de John Kingdon (2003), não apenas avança em termos teóricos propondo a diferenciação entre os conceitos de agenda governamental e agenda decisória, como também propõe, de forma inovadora, um modelo explicativo organizado em torno de fluxos que convergem em determinadas circunstâncias e que seriam responsáveis pelas mudanças na agenda. Outro modelo teórico que busca explicar o processo de formação da agenda foi proposto por Baumgartner e Jones (1993). Por meio do modelo de Equilíbrio Pontuado, os autores demonstraram que há períodos de estabilidade na agenda, marcados pela lógica incremental, bem como momentos que favorecem mudanças rápidas e inesperadas. Buscando entender ambos os processos, os autores avançaram no desenvolvimento teórico propondo a investigação do processo de priorização dos problemas pelos formuladores de políticas, ou seja, a forma como atenção é dirigida a alguns assuntos específicos na agenda (BAUMGARTNER; JONES, 2005) e, mais recentemente, o estudo do processo de definição dos problemas, responsável pela movimentação dos temas pela agenda, considerando o papel da informação (BAUMGARTNER; JONES, 2015).

Partindo desse breve histórico de desenvolvimento da literatura sobre agenda, fica claro que os estudos têm sido desenvolvidos, em grande parte, no contexto dos Estados Unidos. Uma questão relevante consiste no questionamento sobre a aplicabilidade desse referencial em diferentes contextos políticos, administrativos e societários. Nesse sentido, caberia questionar se os modelos teóricos de agenda seriam ferramentas de análise capazes de explicar mudanças nas agendas de diferentes países. Caminhando nessa direção, Baumgartner e Jones (2002) avançam ao expandir os estudos sobre agenda para uma perspectiva teórica sobre o próprio processo de mudança em políticas públicas, propondo o conceito de "dinâmica de políticas públicas" (policy dynamics). A noção de "dinâmica de políticas públicas" é empregada hoje em estudos que buscam identificar as mudanças na atenção governamental - ou seja, movimentações na agenda - em torno de diferentes políticas públicas, durante longos períodos de tempo, e em diferentes sistemas políticos, procurando explicar o processo da mudança em políticas públicas. Na última década, diversos estudos têm sido desenvolvidos em diferentes 
países com base nesse conceito, reunidos numa rede internacional de pesquisa denominada Comparative Agendas Project (CAP). Atualmente, a rede conta com mais de quinze países, entre os quais o Brasil (desde 2015). Esses estudos buscam investigar as agendas governamentais em perspectiva comparada, contribuindo para uma nova etapa nos estudos sobre o processo de formação da agenda governamental. O presente estudo tem como objetivo retomar o histórico dos estudos de agenda no campo da Ciência Política e das Políticas Públicas, destacando as tendências contemporâneas de pesquisa na área e os esforços de elaboração teórico-metodológicos voltados à investigação desse tema. Para tanto identificaremos quatro momentos principais: o início das investigações sobre agenda no campo da ciência política, nos anos 1970; os desenvolvimentos teóricos produzidos no campo de políticas públicas, a partir de meados dos anos 1980 e década de 1990; a expansão dos estudos de agenda, que passam a ser entendidos também como explicações sobre a dinâmica das políticas públicas, ou seja, sobre processos de mudança, nos anos 2000; e o desenvolvimento mais recente, de investigação das dinâmicas das políticas públicas em perspectiva comparada, nos anos 2010.

\section{Anos 1960-1970: Os estudos de agenda-setting entre a comunicação e 0 poder político}

As concepções, modelos e teorias utilizados para os estudos da agenda-setting são múltiplos e foram discutidos em diferentes áreas do conhecimento, sobretudo nos Estados Unidos, a partir da década de 1970. Historicamente, o foco dos estudos sobre o processo formação de agenda tem se constituído, ainda que resumidamente, como o estudo da importância que determinados temas ou problemas assumem em um momento específico. Dada a grande quantidade de acontecimentos, fatos e problemas existentes no dia-a-dia que mereceriam atenção dos atores, de que forma alguns temas são priorizados em detrimento de outros? Essa questão, que envolve um complexo processo de disputa por atenção, resume o principal objetivo nos estudos de agenda: analisar o processo de ascensão e queda na importância de temas ao longo do tempo na agenda de alguns atores e na relação entre eles (SOROKA, 2002).

Rogers e Dearing (1988), ao realizarem um extenso trabalho de identificação e mapeamento dos estudos sobre o processo de agenda-setting, identificaram a existência de três linhas de estudo fortemente desenvolvidas na área: (1) media agenda-setting (agenda midiática);
(2) public agenda-setting (agenda pública); (3) policy agenda-setting (agenda das políticas públicas). De acordo com os autores, ainda que desenvolvidos de forma autônoma e em diferentes áreas do conhecimento, os três tipos de estudos apresentam forte relação teórica e metodológica na análise do processo de priorização de temas e formação da agenda, quer da mídia, da opinião pública ou dos formuladores de políticas governamentais, respectivamente. Existe, no entanto, uma tradição de pesquisa para cada um destes três tipos de agendas que envolve focos analíticos específicos. A primeira, da agenda midiática, apresenta como principal objeto de estudo o processo de seleção dos assuntos que serão noticiados e enfatizados pelos veículos de comunicação de massa (mass media). A segunda tradição, da agenda pública, tem como principal variável a importância percebida pela opinião pública geral sobre um conjunto de temas, ou seja, está relacionada com a identificação dos temas que o público considera importante. A terceira tradição, a agenda de políticas públicas, preocupa-se em estudar o processo de percepção e hierarquização de problemas que chamam a atenção dos formuladores de políticas e grupos próximos a eles.

A primeira tradição dos estudos de agenda-setting está diretamente relacionada ao surgimento e os efeitos da comunicação de massa (mass media) no período pós-Primeira Guerra Mundial. Com a propagação de novos meios de comunicação de massa, os estudos da agenda midiática nascem como uma preocupação voltada para o entendimento do processo de seleção de notícias e, posteriormente, sobre os efeitos que poderiam causar no público. As transformações na velocidade da produção e veiculação de notícias unida à rápida difusão de veículos de comunicação com alta capacidade de alcance de público, começaram a despertar a atenção de pesquisadores. Já em 1922, Lippmann mostrava evidente preocupação sobre o potencial da comunicação de massa, propondo que a mídia tinha o poder de apresentar ideias filtradas para o público, vendo nesses veículos de comunicação uma potente forma de dizer quais questões eram importantes e quais não. Os estudos sobre agenda midiática surgiam, portanto, como um objeto de estudo importante que buscava a compreensão da seleção daquilo que era noticiado e também dos efeitos provocados por essas notícias (WOLF, 1986; TRAQUINA, 1995).

Se num primeiro momento os estudos voltavam-se para a seleção de notícias que seriam veiculadas, com caráter técnico e profissional do campo da comunicação, a preocupação sobre os efeitos dessas notícias na opinião pública assume papel predominante entre os pesquisadores nas décadas seguintes. Entre 1940 a 1960 surgem novos métodos e técnicas de análise de dados. 
Autores importantes desse período, como Lazarfeld, Berelson e Gaudet (1944), Katz e Lazarsfeld (1955), Festinger (1957) e Klapper (1960), intensificaram os estudos da comunicação de massa que buscavam relacionar causa e efeito entre veiculação midiática e resultados eleitorais. Assim, a mídia passa a ser utilizada como uma variável importante para a compreensão do processo de formação da opinião pública. $\mathrm{O}$ mesmo parece acontecer com os estudos da policy-agenda nos quais a agenda midiática passa a ser identificada como sendo uma variável relevante para o processo de reconhecimento e priorização de problemas pelos tomadores de decisão. Segundo Soroka (2002, p. 7),

[...] while public and policy agenda-setting are the most popular lines of agenda-setting research [...] most media agenda-setting observations have taken place as by-products of public and policy agenda-setting studies [...].

A tradição da agenda pública apresenta algumas singularidades quanto ao locus do desenvolvimento dos estudos e os modelos utilizados para a investigação. Foi, sobretudo, na área da comunicação (ou da comunicação política, mais especificamente) que os estudos sobre a public agenda-setting se desenvolveram como área de estudos nos Estados Unidos. Fortemente baseado nos preceitos teóricos de Lippmann (1922) e de Cohen (1963), a tradição dos estudos da opinião pública consolidou-se ao propor uma relação causal direta entre a agenda midiática e a agenda pública. Segundo a hipótese central de Cohen (1963, p. 13)

\section{[...] the press may not be successful much of the time in telling people what to think, but is stunning successful in telling its readers what to think about [...].}

Entretanto, ainda que os referenciais teóricos sobre a opinião pública estivessem surgindo, ou mesmo em processo de amadurecimento em meados dos anos 1960, o conceito de agenda-setting ainda era apenas uma ideia teórica, sem nenhum tipo de aplicação empírica. O próprio nome agenda-setting sequer era reconhecido até a publicação do clássico estudo de McCombs e Shaw (1972) sobre o papel da mídia na campanha presidencial 1968. Conhecido como o "Estudo de Chapel Hill", McCombs e Shaw elaboraram um específico desenho de investigação que pudesse testar a relação entre os assuntos que eram veiculados pela mídia (media agenda-setting) e os assuntos que eram identificados pelo público como sendo assuntos relevantes naquela conjuntura. (public agenda-setting). Baseados na combinação de dois recursos metodológicos - a análise de conteúdo (realizada por meio da análise das reportagens e temas tratados pelos veículos de comunicação de massa) e pesquisas de opinião pública (elaboradas por meio da aplicação de entrevistas a cem eleitores indecisos), os autores comprovaram empiricamente a existência de grande correlação entre os temas noticiados pela mídia e os temas que os entrevistados entendiam como sendo mais importantes. O emblemático estudo de 1972 evidenciou que questões como a política externa, a lei e a ordem e a política fiscal, por exemplo, apareciam com o mesmo grau de importância, dada a frequência com que eram noticiadas ou apontadas como sendo um tema relevante, tanto na agenda midiática quanto na agenda pública. O "Estudo de Chapel Hill" desencadeou uma tradição de pesquisas nos Estados Unidos que buscou analisar as relações existentes entre as agendas midiática e da opinião pública, utilizando uma metodologia mista composta por entrevistas e análise documental.

Desvinculado desta grande tradição de pesquisas sobre opinião pública, é na Ciência Política que surge, paralelamente, ainda no início da década de 1970, os primeiros estudos que formam a linha de pesquisa sobre o policy agenda-process, ou seja, sobre o processo de formação da agenda de políticas públicas. Com foco distinto dos estudos realizados na área da comunicação, a questão-chave para os cientistas políticos não estava na relação entre mídia e opinião pública, mas sim na análise de como uma questão consegue chamar a atenção dos formuladores de política dar origem a uma política pública.

Alimentada, sobretudo, pelos trabalhos sobre o poder e o papel do conflito na política elaborados por Schattscheneider (1960) e Bachrach e Baratz (1962), a tradição de estudos da policy agenda-setting que seria desenvolvida na década seguinte recupera conceitos importantes elaborados por esses autores que tratam do sistema de governança e do reconhecimento de problemas públicos por meio da expansão do conflito. Foco central nos estudos de Schattscheneider é o conceito de "mobilização de opinião" (mobilization of bias), no qual o conflito gerado em torno de um tema (issue), e a organização dos atores em torno da sua definição e promoção, seriam capazes tanto de expandir o conflito transformando-o de uma questão privada em uma questão política, como de suprimi-lo a ponto de o tema em questão não ser reconhecido como um problema. Nas palavras do autor, a política "is the 'socialization' of conflit". (SCHATTSCHENEIDER, 1960, p. 38). Outra importante contribuição do autor que será recuperada pelos estudos da policy agenda-setting está relacionada ao processo de definição e seleção de alternativas. Ainda segundo o autor, os sistemas políticos e seus atores não são capazes de identificar e lidar com todos os problemas existente em uma sociedade, assim como não é possível definir alternativas viáveis para resolver todos os potenciais problemas que possam surgir num dado momento. Essa limitada possibilidade de atuação faz com que o poder fundamental do Estado resida não somente na 
sua capacidade de identificar e priorizar problemas, mas também na proposição de alternativas para solucioná-los. É a união entre identificação de problema e proposição de alternativas que geram decisões e a ações políticas. Schattschneider (1960, p. 66) diz que

\section{[...] the definition of alternatives is the supreme instrument of power.}

Bachrach e Baratz (1962) ampliam a discussão inicialmente formulada por Schattschneider acerca do processo de mobilização e expansão do conflito ao proporem a existência das "duas faces do poder" (two faces of the power). Retomando conceitos centrais do processo de influência da teoria pluralista, os autores chamam atenção para o processo de bloqueio na identificação e reconhecimento de problemas. Se por um lado existe o reconhecido processo de coerção que entende a habilidade de " $A$ " fazer com que " $B$ " tome decisões e ações que sozinho ele não faria, também existe o outro lado da moeda, até então não estudado, que implica em dizer que "A" pode agir de forma a fazer com que "B" continue fazendo aquilo que ele já está fazendo, sem se afetar a possíveis alternativas que acarretem em mudanças.

To the extent that $A$ succeeds in doing this, $B$ is prevented, for all practical purposes, from bringing to the fore any issues that might in their resolution be seriously detrimental to A's set of preferences. BACHRACH; BARATZ, 1962, p. 952).

As ideias trazidas por esses autores da Ciência Política estadunidense no início dos anos 1960 contribuíram para a criação, uma década depois, de uma nova tradição nos estudos do processo de agenda-setting (SOROKA, 2002; BIRKLAND, 2005).

\section{Anos 1970: participação, agendas e 0 ciclo de atenção}

O termo policy agenda-setting é empregado, pela primeira vez, pelos autores Cobb e Elder (1971, p. 905), que direcionam seus estudos para a relação entre a formação da agenda de políticas públicas (como ela é definida, quais temas são considerados) e a participação de diferentes atores no policymaking process (quem participa? como participa?):

We are raising the basic questions of where public-policy issues come from. We are concerned with how issues are created and why some controversies or incipient issues come to command the attention and concern of decision makers, while others fail. In other words, we are asking what determines the agenda for political controversy within a community. How is an agenda built (i.e. how is an issue placed on it) and who participates in the process of building it?
Os estudos de Cobb e Elder (1972) estabelecem uma relação direta entre o processo de formação da agenda governamental e a ampliação da democracia via participação popular no processo de políticas públicas. Essa relação parte de dois grandes pressupostos. O primeiro entende que os problemas que demandam ação governamental são socialmente construídos e filtrados pelos tomadores de decisão que priorizam alguns problemas em detrimento de outros. Desta forma, expandir a identificação de uma demanda envolvendo a participação de mais atores, captando a atenção da população, aumentaria tanto o escopo democrático via participação popular no reconhecimento e expansão de um problema, quanto influenciaria na dinâmica de priorização desses problemas uma vez que a mobilização popular tornaria essas questões mais visíveis ao Estado. O segundo pressuposto, identificado como uma importante inovação nos estudos de agenda-setting, aponta para a existência de diferentes níveis de agenda, como as agendas sistêmica e institucional. A agenda sistêmica, para os autores, compreende o conjunto das questões consideradas pelos participantes do processo político. De acordo com Cobb e Elder (1972, p. 85):

\section{The systemic agenda consists of all issues that are commonly perceived by members of the political community as meriting public attention and as involving matters within the legitimate jurisdiction of existing governmental authority. Every local, state, and national political community will have a systemic agenda.}

As questões que estão contidas na agenda sistêmica podem ser identificadas como aquelas que despertaram a atenção da opinião pública. A partir do momento em que um grupo ou parte considerável do público passa a demandar algum tipo de ação concreta com relação ao problema identificado, ou ainda no caso de haver uma percepção pelos membros de uma comunidade política de que uma questão deve ser tratada pelo governo, passamos a identificá-la como parte da agenda sistêmica. Entretanto, conforme a proposta dos autores, nem todas as questões na agenda sistêmica se tornarão alvo de políticas públicas. Somente algumas dessas questões conseguirão chamar a atenção do governo e, ao serem priorizadas, passarão a um tipo de agenda, chamada de agenda institucional/governamental/formal:

The institutional, governmental, or formal agenda may be defined as that set of items explicitly up for the active and serious consideration of authoritative decision-makers. Therefore, any set of items up before any governmental body at the local, state, or national level will constitute an institutional agenda. (COBB; ELDER, 1972, p. 85). 
A grande questão e foco dos trabalhos na ciência política reside exatamente na compreensão do movimento que faz com que um tema da agenda sistêmica passe para a agenda governamental. Cobb et al. (1976) propõem a existência de três modelos distintos pelos quais as questões presentes na agenda sistêmica passam a integrar a agenda governamental. Entendida como o modo predominante de ascensão à agenda governamental em sociedades liberais e igualitárias, o modelo "outside initiative model" se aplica a situações em que um grupo fora da estrutura do governo articula uma questão/problema e tenta expandir o interesse desse assunto para outros grupos, buscando mobilizar a população para ganhar um lugar na agenda governamental.

A segunda forma de articulação entre agenda sistêmica e agenda governamental analisada pelos autores é a "mobilization model". Diferente do modelo anterior, em que a questão nasce por fora do governo, esse modelo evidencia que questões também são originadas dentro do próprio governo. "The mobilization model consider issues which are initiated inside government and consequently achieve formal agenda status almost automatically" (COBB et al., 1976, p. 127-128). Uma vez nascendo no próprio governo, a mobilização aqui se faz necessária como uma justificativa de ação. No sentindo inverso, é o governo que chama a atenção do público para um dado problema na busca de maior efetividade na implementação de uma política.

A terceira relação entre as agendas sistêmica e governamental identificada por Cobb et al. (1976) (inside initiative model) assim como a anterior, também se origina dentro governo. São questões identificadas pelos formuladores de políticas mas que, diferente do modelo anterior, não nascem necessariamente na sistêmica. Este modelo, mais vinculado com decisões técnicas, surge em órgãos governamentais mais específicos que buscam limitar que as decisões caiam na agenda sistêmica onde podem encontrar limitações e oposição. De acordo com os autores, "Inside initiative model describe issues which arise within governamental sphere and whose supported do not try to expand them to mass media." (COBB et al., 1976, p. 128).

Outra contribuição importante para a o estudo da formação da agenda foi desenvolvida por Downs (1972) que defende a existência de um ciclo de atenção responsável por influenciar o comportamento do público em relação aos problemas e, consequentemente, sobre a agenda. $\mathrm{O}$ modelo do ciclo de atenção leva em consideração uma importante característica da opinião pública sobre sua percepção de problemas e políticas públicas: sua natureza vaga e transitória. Para Downs, o processo de formação de políticas públicas depende da atenção momentânea dada pela opinião pública à problemas e questões sociais. Assim, o "issue attention cycle" pretende explicar a oscilação na atenção da opinião pública sobre a percepção de problemas públicos. O ponto de partida desse ciclo reside no momento em que um determinado assunto, por diversos motivos, passa a ser entendido como um problema e torna-se foco de atenção popular, permanecendo em destaque e em debate por durante um breve período de tempo. O ciclo transforma-se quando o assunto é modificado, e outro tema ou problema passa a ser centro da atenção pública, ocupando o espaço do assunto anterior, mesmo que este não sido resolvido (DOWNS, 1972). Para melhor compreensão, é possível separar o ciclo em cinco fases: (a) "pré-problema”, na qual uma condição social existente ainda não chama a atenção do público; depois passando a (b) um momento de "descoberta e entusiasmo", na qual o público se torna consciente de uma questão e alarmado com suas consequências; seguindo para um momento de (c) percepção sobre as dificuldades e os custos envolvidos na resolução do problema; até que (d) o interesse do público sobre a questão começa a declinar gradualmente; e finalmente, (e) saída do problema do centro das preocupações do público, seja por meio da substituição do problema por outro ou seu deslocamento para fora do debate público, onde recebe um nível muito menor de atenção.

$O$ resultado desses problemas percebidos resultaria em demandas por parte da população por ação governamental para solucioná-los. De acordo com o autor:

[...] a atenção do público raramente permanece firmemente focada sobre qualquer questão doméstica por muito tempo - mesmo quando envolve um problema de importância crucial para a sociedade. Em vez disso, um "ciclo de atenção a questões" sistemático parece influenciar fortemente as atitudes e comportamento do público em relação à maioria dos problemas domésticos centrais. Cada um desses problemas repentinamente surge como predominante, assim permanece por algum tempo e então - ainda que não resolvido - gradualmente desaparece do centro da atenção pública. (DOWNS, 1972, p. 42)

Ainda segundo o autor, algumas características podem fazer com que alguns problemas ganhem atenção da opinião pública. Entre essas peculiaridades, estão os problemas que afetam uma minoria da população; aqueles problemas resultados de arranjos institucionais ou políticas que beneficiam uma maioria da população, ou mesmo problemas capazes de gerar momentos "dramáticos", mas não períodos contínuos de atenção, como, por exemplo, desastres naturais causados por chuvas em períodos específicos do ano, ou a proliferação de uma doença sazonal. 


\section{Anos 1980-1990: em busca de explicações para mudanças rápidas nas agendas}

Os estudos sobre o processo de produção de políticas, desenvolvidos em meados dos anos 1980 e 1990 nos Estados Unidos, deram novo fôlego às investigações sobre a agenda. Esses estudos mudaram o foco até então dominante nas investigações, que se desenvolviam em torno das relações entre a agenda sistêmica (as questões e problemas que despertam a atenção do público) e governamental (questões e problemas seriamente considerados pelos policy-makers) (COBB; ELDER, 1972). Os estudos que deram continuidade à investigação do processo de agenda-setting passaram a se concentrar nos intrincadas relações entre diferentes variáveis explicativas para a formação da agenda governamental buscando mostrar que, mesmo dentro desse limitado conjunto de questões que emergem à consideração dos policymakers, há ainda diversos processos seleção. Ou seja, nem todas as questões seriamente consideradas pelos tomadores de decisão - e que integram a agenda governamental - tornar-se-ão políticas públicas. Algumas serão consideradas e posteriormente descartadas, enquanto outras seguirão seu curso pelo competitivo processo de produção de políticas.

Essa mudança de perspectiva fica clara observando o modelo de Múltiplos Fluxos, de John Kingdon (2003) . Nesse estudo, o autor não apenas avança na investigação do processo de agenda-setting em termos teóricos, propondo a diferenciação entre os conceitos de agenda governamental e agenda decisória, como também propõe, de forma inovadora, um modelo explicativo organizado em torno de fluxos que convergem em determinadas circunstâncias e que seriam responsáveis pelas mudanças na agenda. A agenda governamental é entendida como o conjunto de assuntos sobre os quais o governo (e pessoas ligadas a ele) concentram sua atenção num determinado momento (KINGDON, 2003, p. 3). Já a agenda decisória consiste num subconjunto da agenda governamental que contempla questões prontas para a tomada de decisão pelos formuladores de políticas, ou seja, prestes a se tornarem políticas públicas (KINGDON, 2003, p. 4).

Esta diferenciação se faz necessária, segundo o autor, porque ambas as agendas são afetadas por processos diferentes. $\mathrm{O}$ autor argumenta que mudanças na agenda governamental resultam da combinação de dois fatores. O primeiro é a existência de um problema capaz de chamar a atenção dos tomadores de decisão (fluxo de problemas). Tais problemas, para Kingdon, não são o resultado de indicadores objetivos, e sim representações sociais. Por meio de um processo de seleção e interpretação de indicadores (como dados relativos ao monitoramento de programas governamentais, relatórios de desempenho de ações, entre outros), os problemas são definidos e passam a ter maiores ou menores chances de chamar a atenção dos tomadores de decisão. Da mesma forma, grandes eventos (desastres, catástrofes, crises, acidentes de amplas proporções), contribuem para focalizar a atenção sobre uma questão, mas não transformam essas questões automaticamente em problemas. A interpretação desses eventos e seu entendimento enquanto problemas que demandam ação governamental é que determina o sucesso de uma questão na agenda governamental. $\mathrm{O}$ segundo fator fundamental para o acesso de uma questão à agenda governamental é um momento político favorável (fluxo político). Esse processo relaciona-se com a opinião pública (percepção geral da sociedade sobre determinadas questões e sobre encaminhamentos específicos), a receptividade das forças políticas organizadas em torno de determinadas soluções (posicionamento dos grupos de interesse em relação a uma questão, por exemplo) e mudanças no próprio governo (mudança de pessoas em posições estratégicas dentro da estrutura governamental, mudanças de gestão, mudanças na composição do Congresso, entre outros fatores).

A mudança na agenda governamental, portanto, resulta da combinação desses dois fatores - a forma pela qual um problema é percebido (fluxo de problemas) e as alterações na dinâmica política e da opinião pública (fluxo político). Porém, no que diz respeito à mudança na agenda decisória, além desses dois fatores, um terceiro torna-se fundamental: o conjunto de alternativas disponíveis (fluxo de alternativas ou soluções). As alternativas são desenvolvidas por especialistas (pesquisadores, assessores parlamentares, acadêmicos, funcionários públicos, analistas pertencentes a grupos de interesses, entre outros) e não são, necessariamente, relacionadas à percepção de problemas específicos. Considerando os dois níveis de agenda, tal como definidos por Kingdon, pode-se dizer que uma política pública só tem início se uma questão chega à agenda decisória, depois de passar pela agenda governamental. Para explicar esse processo, o modelo prevê o surgimento de alguns momentos críticos (as chamadas "janelas de políticas", ou policy window), nos quais a oportunidade de conexão entre os três fluxos é maior. Quando tais "janelas de oportunidade política" se abrem, entram em cena os empreendedores de políticas, indivíduos que podem fazer parte do governo (no Poder Executivo, ocupando altos postos ou em funções burocráticas; no Congresso, como parlamentares ou assessores), ou não (participando de grupos de interesse, da comunidade acadêmica, da mídia, por exemplo). Estes 
indivíduos reconhecem o momento da oportunidade, seu caráter transitório, e agem no sentido de conectar os três fluxos, promovendo o acesso de uma questão à agenda decisória.

Outro modelo teórico que busca explicar o processo de formação da agenda foi proposto por Baumgartner e Jones (1993). Para os autores, a explicação sobre mudanças na agenda está relacionada à tese do Equilíbrio Pontuado, que procura explicar longos períodos de estabilidade nos sistemas políticos, pontuados por momentos de mudanças profundas e rápidas. Por meio da investigação de alguns casos no contexto do sistema político dos Estados Unidos (energia nuclear, fumo e tabaco, pesticidas, entre outras) e considerando períodos relativamente prolongados de tempo, os autores observaram a dinâmica dos subsistemas, os quais denominaram "monopólio de políticas". Esses monopólios são arranjos institucionais baseados em um conjunto de entendimentos sobre uma determinada questão e a partir do qual os atores ganham a habilidade de controlar a interpretação sobre um problema e a forma como este é percebido e discutido. Os monopólios permitem que o processo decisório seja limitado a um grupo pequeno de atores, restringindo o acesso dos demais e, dessa forma, produzem e mantém a estabilidade na produção de políticas públicas, retingindo o acesso de novas questões à agenda.

Assim, o modelo de Equilíbrio Pontuado procura explicar períodos de equilíbrio na produção de políticas, que se mantém enquanto uma questão é dominada por um monopólio, e que se rompe quando a questão escapa ao controle do monopólio e chega à agenda do macrossistema. Uma vez no macrosistema, pequenas mudanças podem ser amplificadas, transformando completamente a política. Nesse caso, tem-se o que os autores denominam feedback positivo: um processo que ocorre ao longo do tempo, envolvendo uma série de eventos relacionados a uma política pública paralelamente à mudanças amplas, rápidas e geralmente inesperadas. Por outro lado, o feedback negativo envolve os processos relacionados à estabilidade, produzida pelos monopólios. Os monopólios reforçam dois elementos: estruturas institucionais que limitam o acesso de participantes no debate sobre uma política e imagens (policy images), compostas por ideias e entendimentos a respeito de uma política e que restringem a forma como uma questão é debatida. Dessa forma, o processo de feedback negativo se produz em estruturas institucionais dominadas por ideias estáveis e essa combinação pode se manter durante décadas, inibindo a mudança. Debruçados sobre os casos selecionados para análise, os autores mostraram que quando esses monopólios se romperam, o sistema tornou-se propenso à mudança, facilitando o surgimento de novas questões na agenda (feedback positivo).

Um bom exemplo é o estudo sobre a construção de um monopólio em torno da política de energia nuclear desenvolvida nos Estados Unidos (BAUMGARTNER; JONES, 1993). Ao longo das décadas de 1940 e 1950, essa política esteve associada a uma imagem muito positiva sobre as questões nucleares, envolvendo elementos como a característica limpa dessa fonte de energia e a possibilidade de redução da poluição, o aspecto econômico da produção de energia impactando diretamente na redução das contas para os consumidores, a alta tecnologia envolvida e o desenvolvimento de uma fonte de energia de baixo custo para o futuro, reduzindo a dependência de outros países para obtenção de petróleo, entre outros aspectos. É certo que outras imagens sempre estiveram presentes, envolvendo aspectos negativos como o potencial altamente destrutivo das armas nucleares, interesses de empresas privadas, mutações genéticas, entre outros entendimentos, mas a visão positiva era, nesse período, muito mais presente. Membros do governo, da comunidade científica, jornalistas, e empresas do setor privado estavam entre os atores que compartilhavam essa visão positiva. Além da imagem positiva, mudanças institucionais foram produzidas e permitiram aos defensores desse entendimento sobre a energia nuclear exercer controle sobre a política. A Comissão de Energia Atômica, criada em 1946, tinha a responsabilidade de promover a regulação e a promoção da produção nuclear, bem como de produzir os itens militares associados à energia nuclear. Além da Comissão, integraram o monopólio os construtores das centrais nucleares, a elite tecnocrática civil e militar, e a Comissão Mista sobre Energia Atômica, comitê conjunto entre a Câmara e o Senado. Segundo os autores, o monopólio manteve-se enquanto a imagem positiva perdurou e o controle sobre a questão permaneceu nas mãos da Comissão de Energia Atômica e da Comissão Mista. Duas décadas depois, nos anos 1960 e 1970, o monopólio entrou em colapso. Vários fatores contribuíram a dissolução desse monopólio. No interior da Comissão de Energia Atômica, diversos membros questionavam sobre aspectos relacionados à segurança, levando à deserção de parte do grupo original. A questão se expande para além dos limites do monopólio e da Comissão de Energia Atômica quando cientistas compartilham as preocupações internas com segurança com outras organizações, previamente excluídas do debate e também relacionadas à temática. A imagem passa de positiva à negativa também na mídia, que destaca elementos até então ausentes, como preocupações com a segurança e com possíveis impactos negativos ao 
meio ambiente e cristaliza-se com o acidente em Three Mile Island, no estado da Pennsylvania, ocorrido em 1979. Paralelamente, novos atores passaram a demandar o controle sobre a questão da energia nuclear, o que pode ser observado com o aumento do número de comitês e subcomitês no Congresso voltados a ações de controle da energia nuclear. Em 1974 a Comissão de Energia Atômica foi desmantelada e suas funções regulatórias atribuídas à Comissão Regulatória Nuclear, e as demais funções direcionadas para Agência de Pesquisa e Desenvolvimento Energéticos. Assim, o monopólio em torno da política nuclear - envolvendo tanto os entendimentos a respeito da questão quanto as instituições construídas para dar-lhe suporte - foi destruído. Nas décadas seguintes, a imagem negativa em torno da questão permaneceu e até recentemente a energia nuclear não consistia em uma opção seriamente considerada pelos tomadores de decisão.

Assim, durante as décadas de 1940 e 1950 a política foi caracterizada pela estabilidade (feedback negativo), garantida por um entendimento comum e estruturas institucionais capazes de fortalecer o monopólio. A partir das décadas de 1960 e 1970, a questão passa a escapar ao controle do monopólio, envolvendo diferentes ideias e controles institucionais e tornando a política nuclear propensa a mudanças (feedback positivo). Dessa forma, o modelo de Equilíbrio Pontuado propunha explicações para as mudanças na agenda verificadas nos monopólios de políticas vinculados aos casos selecionados para a investigação (BAUMGARTNER; JONES, 1993).

\section{Anos 2000: agendas e a dinâmica das políticas públicas}

Nas décadas posteriores aos trabalhos de Kingdon (2003) e Baumgartner e Jones (1993), os estudos sobre agenda no campo de políticas públicas caracterizaram-se pela disseminação desses modelos teóricos e pela realização de testes empíricos em diferentes áreas de políticas ${ }^{1}$. Em termos do desenvolvimento das formulações teóricas, cabe destacar alguns aprimoramentos realizados pelos autores vinculados ao modelo de Equilíbrio Pontuado. De forma geral, as preocupações iniciais sobre a investigação das agendas avançaram para explicações sobre a dinâmica das políticas e os processos de mudanças em políticas públicas.

Os autores aprofundaram a investigação de processos de estabilidade e mudança por meio

\footnotetext{
Para uma visão geral sobre as pesquisas empíricas realizadas por diversos pesquisadores na literatura internacional, ver o texto de Zahariadis sobre o modelo de Múltiplos Fluxos, e o artigo de Baumgartner, Jones e Mortensen sobre o modelo de Equilíbrio Pontuado. Ambos os estudos foram publicados em Sabatier e Weible (2014).
}

de uma análise mais detalhada dos processos de feedback positivo e negativo (BAUMGARTNER; JONES, 2002). Tais conceitos, presentes nos estudos iniciais sobre agenda (BAUMGARTNER; JONES, 1993) são expandidos e empregados para explicar o funcionamento do sistema político norte-americano como um todo, e não apenas a dinâmica de subsistemas com as características dos monopólios de políticas. Para os autores, o feedback positivo - mudanças rápidas, abruptas e muitas vezes imprevisíveis - tem raízes em dois processos: mimetização e deslocamento de atenção. $\mathrm{O}$ processo de mimetização entra em cena quando indivíduos, em situações de tomada de decisão, observam o comportamento de outros e agem de acordo. Esse processo, denominado pelos autores de efeito "cascata social", explica mudanças no comportamento dos atores, derivado de alterações em seus valores e ideias, muitas vezes de maneira independente de eventos externos que poderiam ser associados à mudança em uma política. $\mathrm{O}$ segundo processo relacionado ao feedback positivo - o deslocamento de atenção - baseia-se na premissa do processamento serial: em contextos complexos de tomada de decisão, os indivíduos não têm condições de prestar atenção a todas as dimensões da escolha simultaneamente. Nessas situações, os indivíduos consideram uma ou mais dimensões para embasar sua decisão. Assim, apenas alguns atributos presentes nas questões que envolvem as políticas públicas (comumente multidimensionais e complexas) são considerados. Juntamente com o processo de mimetização, o deslocamento de atenção é um elemento fundamental para a compreensão do processo decisório governamental:

Public debates [...] are simultaneously subject to
the processes of attention shifting common to all
multidimensional and complex decisions and also
to the cascading and mimicking phenomena that we
have noted where decisions are made in reaction to
the decisions of those around oneself. The result of
these twin characteristics of policy debates is that
positive feedback is a fundamental feature of many
policies. (BAUMGARTNER; JONES, 2002, p. 20).

Um exemplo claro da aplicação dos modelos de análise de políticas públicas pode ser visto no estudo de caso sobre a construção e destruição do monopólio na política de telecomunicação estadunidense entre os anos de 1960 e 1990. De acordo com McLeod (2002), os estudos demonstram tanto períodos de estabilidade quanto uma clara e profunda mudança na atenção institucional à questão do monopólio na política de telecomunicação. A análise proposta pelo autor envolve atores e instituições reguladoras, como o FCC (Federal Communications Commission); o Congresso Nacional dos Estados Unidos; a AT\&T (até então a 
única provedora de telefonia fixa nos Estados Unidos); além de atores e grupos de pressão importantes para a mudança sobre a forma de prestação dos serviços de telecomunicação no país. De acordo com esse estudo (que elabora uma análise longitudinal-temporal) entre os anos 1930 e 1994, o período que engloba os anos 1930 a meados de 1960 é marcado por uma lógica incremental, de estabilidade, e pequenas mudanças na atenção e transformações na política de telecomunicações. Entretanto, as decisões e posicionamentos da FCC no final de 1960 e início de 1970 iniciou modificações na construção da imagem da política enviando sinais tanto para atores governamentais quanto para grupos de interesse que aquele poderia ser o momento certo para questionar e propor mudanças no atual desenho que conferia à AT\&T o monopólio na prestação de serviço de telefonia nos Estados Unidos.

A FCC foi, portanto, a entidade responsável pelo início das mudanças com relação ao modelo de monopólio da empresa AT\&T em meados da década de 1970. Em seguida, após as propostas de alteração tomarem o cenário público, o Congresso foi envolvido, no final de 1970. O curso histórico dessa política não apresentou enfrentamento ou fortes impeditivos de outras instituições políticas envolvidas na política de telecomunicações, proporcionando um aumento progressivo na atenção institucional à proposta em questão. Este aumento da atenção institucional, quando analisado pelo modelo teórico do Equilíbrio Pontuado, evidencia aquilo que os autores chamam de um "indicativo do período de feedback positivo", ou seja, indicadores de mudança da agenda governamental em torno de um tema ou política específica. A quebra do monopólio só ocorreu após a atuação conjunta de grupos de interesse até então excluídos da formulação das políticas de telecomunicações e de membros de instituições de regulação, como a FCC, que estavam no controle da política de telecomunicações. $\mathrm{O}$ avanço e as transformações na tecnologia são tidos pelo autor como um aspecto importante que levou a um aumento do conjunto de atores que se opunham ao modelo de monopólio, o que levou à destruição do monopólio da AT\&T no início doa anos 1980.

De forma similar, Valerie Hunt (2002) elabora um estudo sobre as mudanças na atenção do Senado e da Câmara com relação à política de imigração e sua consequente reforma. A autora analisa o período entre 1947 e 1993 e as transformações na atenção dos tomadores de decisão em torno da reforma da política de imigração estadunidense. Para tanto, a base de dados utilizada pela autora para verificar tais mudanças é composta pelos dados do Policy Agendas Project-audiências do Congresso; publicação de leis, e o Congressional Quarterly Almanac. Uma segunda fonte de dados utilizada foram os Estatuto de imigração dos Estados Unidos. O resultado encontrado por esse estudo mostra que, considerando as atividades das Audiências da Câmara e do Senado entre 1965-1993, o período foi marcado por ações de mobilização e contra-mobilização em relação à reforma da política de imigração entre e dentro das duas Casas. A Câmara inclinava seus esforços para manter as medidas de reforma de imigração fora da agenda, utilizando-se de vários processos institucionais. Já o Senado, reagindo ao posicionamento contrário da Câmara, apresentou-se de forma instável no período analisado, evidenciando dramáticas oscilações ascendentes e descendentes na entrada do tema em sua agenda. As mudanças somente ocorreram com a inserção de novos atores e grupos de pressão que, sob o argumento da necessidade de uma oferta de trabalho barato e estável, defendiam a redefinição da política de imigração. Esta nova onda de mudanças no entendimento da questão da legalidade e processos de imigração finalmente resultou em uma solução política que concedeu anistia aos estrangeiros em situação irregular que tinha residido nos EUA desde 1982, garantindo assim, e sobretudo, a mão de obra necessária aos empregadores estadunidenses. Este estudo da reforma da política de imigração dos EUA demonstra que a multidimensionalidade de questões cria oportunidades para grandes modificações na definição de um problema e em suas soluções possíveis dentro de um subsistema de política. A aplicação do modelo do Equilíbrio Pontuado evidencia que a interação de temas, atores e instituições distintas pode levar a mudanças significativas em políticas públicas.

\section{Anos 2010: agendas e a dinâmica das políticas públicas em perspectiva comparada}

O desenvolvimento mais recente dos estudos de agenda está relacionado à aplicação da ideia de "dinâmica de políticas públicas" em estudos comparativos (BAUMGARTNER; JONES; WILKERSON, 2011), ou seja, investigações sobre o processo de mudanças em políticas em diferentes contextos nacionais. Estudos recentes buscam identificar mudanças na atenção governamental - ou seja, movimentações na agenda - em torno de diferentes políticas públicas, durante longos períodos de tempo, e em diferentes sistemas políticos. Esses estudos procuram mostrar como novas ideias, novos entendimentos sobre as questões e problemas são aceitos (ou não) em diferentes contextos nacionais. Considerando que a literatura sobre agenda, desde os anos 1970, tem sido produzida no contexto dos Estados Unidos, essa tendência recente tem contribuído para 
ampliar a compreensão dos limites e possibilidades das teorias sobre agenda e mudança em políticas públicas em diversos países (BAUMGARTNER; GREEN-PEDERSEN; JONES, 2006, p. 967).

Um achado importante desses estudos aponta para a viabilidade das explicações sobre estabilidade e mudança em políticas públicas baseadas nas formulações de Baumgartner e Jones (1993, 2005, 2015) em diferentes sistemas políticos. Apesar das diferenças, verificou-se dinâmicas de mudanças em políticas públicas com as características pontuações em diferentes países (BAUMGARTNER; GREEN-PEDERSEN; JONES, 2006; BAUMGARTNER; JONES; MORTENSEN, 2014). Os pesquisadores estão reunidos numa rede internacional denominada Comparative Agendas Project (CAP, 2015) que conta com estudos sobre as diferentes realidades das mudanças em políticas públicas no Canadá, Bélgica, Dinamarca, Inglaterra, França, Holanda e também a União Europeia ${ }^{2}$. Esse novo momento dos estudos de agenda-setting no campo de políticas públicas, envolvendo a análise comparada das agendas de diferentes países, ainda está em desenvolvimento e enfrenta uma série de desafios que envolvem a definição de uma estrutura de códigos comum para os diversos contextos políticos-institucionais, o desenvolvimento de categorias válidas do ponto de vista comparativo, testes de resultados, entre outras questões de ordem metodológica. Apesar desses desafios, os autores têm apontado que o arcabouço teórico e metodológico do Comparative Agendas Project é aplicável a qualquer sistema político e que permite a comparação entre agendas de diferentes países (JOHN, 2006).

Entre os mais variados estudos que foram produzidos pelo CAP, podemos ilustrar alguns casos produzidos para explicar a estabilidade e a mudança na agenda governamental de países integrantes do grupo como a Inglaterra (JOHN, 2006) e a análise do impacto da mídia, da opinião pública e da violência política sobre os orçamentos urbanos. Neste estudo, os autores procuram explicar as relações existentes entre as drásticas mudanças nas dotações orçamentárias nacionais aplicadas às políticas urbanas na Inglaterra ao longo do período compreendido entre 1966-2003. A análise, que se utiliza do modelo proposto pelo CAP, encontra explicações sobre a mudança no orçamento ao estabelecer relações deste com a atenção da mídia para as questões urbanas e com o mapeamento da opinião pública sobre as questões como o desemprego, o controle partidário, e as questões econômicas.

\footnotetext{
2 O resultado desses estudos foram reunidos e publicados em "Journal of European Public Policy" (JOURNAL..., 2006).
}

No estudo de caso elaborado pelo grupo francês (BAUMGARTNER; FOUCAULT; FRANÇOIS, 2006) a análise é feita sob o lócus dos processos orçamentários da França com o intuito de verificar tanto a existência de momentos de estabilidade e mudanças incrementais, quanto momentos de rápida e profunda mudança na designação orçamentária francesa. Os dados incluem o orçamento geral do Estado desde 1820; orçamentos ministeriais de sete ministérios distintos, desde 1868; e uma série mais completa cobrindo dez ministérios desde 1947.

O CAP também conta com estudos supranacionais, como é o caso da investigação sobre a formação da agenda na União Europeia (PRINCEN; RHINARD, 2006). Os autores identificaram a existência de dois tipos de processos de formação da agenda na União Europeia: um processo "cima para baixo", operado por instituições políticas de alto nível que incitam a ação da UE; e outro "de baixo para cima" por meio da orientação de especialistas em políticas específicas e grupos de trabalho. Como resultado, o artigo mostra como esses dois processos, teoricamente opostos, podem interagir e se entrelaçar no curso de processos reais de definição da agenda governamental. Para tanto, a abordagem teórica é demonstrada por meio de dois estudos de casos contrastantes: um da política anti-tabagismo da UE e uma das políticas anti-bioterrorismo também da UE.

Além da expansão para a análise de diferentes países e sistemas políticos, e até para sistemas supranacionais, como é o caso descrito acima, os estudos avançam também na direção de uma nova abordagem dos estudos da política pública em perspectiva comparada que busca analisar a forma como a definição de problemas, a escolha de alternativas e o próprio processo de formação da agenda governamental ganha a atenção dos tomadores de decisão e produz diferentes políticas públicas em distintos contextos nacionais. Considerando que os estudos de política comparada existentes tendem a se concentrar em explicar como diferenças institucionais ou programáticas afetam a produção de políticas, a abordagem do CAP sugere que o processo de definição de problemas afeta a produção de políticas públicas, suas instituições e atores. Dessa forma, nota-se o surgimento de estudos comparados elaborados por meio de uma base de dados e de uma metodologia comum, como pode ser notado na abordagem comparativa sobre o uso de pesticidas no Canadá e nos EUA (PRALLE, 2006) e no estudo sobre formulação de políticas de saúde em perspectiva comparada entre Dinamarca e Estados Unidos (GREEN-PEDERSEN; WILKERSON, 2006). 


\section{Considerações finais}

Este artigo buscou realizar um mapeamento histórico sobre as transformações nos estudos de agenda governamental ao logo das últimas décadas. Iniciamos nossa trajetória recuperando os trabalhos entre as áreas da Ciência Política e da Comunicação que se dedicaram aos estudos do poder político e da formação da agenda midiática nos anos 1960. No campo da Ciência Política, os primeiros estudos realizados por Cobb e Elder (1971, 1972), caracterizaram-se por uma forte relação entre o processo de formação da agenda governamental e a ampliação da democracia via participação popular. Marcado, sobretudo, pelo debate advindo da Ciência Política tradicional, a grande inovação desses estudos está na diferenciação entre a agenda sistêmica e a agenda governamental. O crescimento da temática é fortalecido pelos estudos de Anthony Downs (1972) acerca do ciclo de atenção e da relação existente o papel e o funcionamento da opinião pública para a emergência de temas e formulação de políticas públicas. A segunda fase (1980-1990) é marcada pelo surgimento de importantes modelos teóricos que buscaram analisar não somente os momentos de mudanças pequenas e graduais (incrementalismo), mas também os momentos de rápida e significativa mudança na agenda governamental. Os modelos de Múltiplos Fluxos de John Kingdon (2003) e de Baumgartner e Jones (1993) surgem, então, como as principais inovações teóricas e metodológicas nos estudos da formação e da mudança da agenda governamental nos Estados Unidos. A terceira fase é compreendida pela difusão desses modelos e sua aplicação em diversos casos, sobretudo em políticas estadunidenses. A consolidação dos modelos e os ajustes realizados durante o final dos anos 1990 e início dos anos 2000 caminharam para a experimentação empírica na análise do processo de formação das agendas e para explicações mais sistemáticas sobre a dinâmica das políticas e seus processos de mudanças. A quarta e mais recente fase dos estudos apresenta tanto avanços metodológicos quanto a superação de limites territoriais com a ampla difusão dos modelos para análises dos mais distintos sistemas políticos ao redor do globo. A criação do CAP - Comparative Agendas Project - que conta com a participação de mais de quinze países de quatro continentes diferentes marca o início da década de 2010 como um momento de avanço nos estudos comparados, baseados na adoção de uma metodologia comum.

No Brasil, apesar de os estudos sobre as políticas públicas vivenciarem um período de expansão nas últimas décadas (HOCHMAN; ARRETCHE; MARQUES, 2007; MARQUES; FARIA, 2013), é possível perceber que o campo, marcado por uma multiplicidade de estudos de caso de políticas setoriais específicas, geralmente publicados em teses e dissertações, muitas vezes desvinculado do debate e da literatura internacional, ainda carece de reflexões teóricas mais aprofundadas (ARRETCHE, 2003; FARIA, 2003; SOUZA, 2006; FARAH, 2013; CAPELLA, SOARES; BRASIL, 2014). Um passo importante nessa direção foi dado no início de 2015 com a inclusão do "Laboratório de Agenda Governamental"3 (Brazilian Policy Agendas Project) no grupo de países pertencentes ao CAP. Esta iniciativa, que pretende monitorar e analisar o ingresso e saída de temas na agenda governamental, tendo como foco principal o governo federal brasileiro, insere o Brasil no mapa dos estudos interacionais, principalmente voltado para a quarta fase, marcada pela difusão internacional e pelos estudos comparados. A investigação conduzida no Brasil pretende lançar luzes sobre os processos de formação da agenda brasileira, por meio do mapeamento dos níveis de atenção governamental às diversas políticas públicas ao longo do tempo. Além disso, ao mesmo tempo em que a pesquisa pode beneficiar-se de uma perspectiva comparada, pode também auxiliar a identificar limites e potencialidades das teorias mais recentes sobre a formação da agenda.

\section{Referências}

ARRETCHE, M. T. S. Dossiê agenda de pesquisas em políticas públicas. Revista Brasileira de Ciencias Sociais, v. 18, n. 51, p. 7-10, 2003.

BACHRACH, P.; BARATZ, M. S. Two faces of power. The American Political Science Review, v. 56, n. 4, p. 947-952, 1962.

BAUMGARTNER, F. R.; FOUCAULT, M.; FRANÇOIS, A. Punctuated equilibrium in French budgeting processes. Journal of European Public Policy, v. 13, n. 7, p. 1086-1103, 2006.

BAUMGARTNER, F. R.; GREEN-PEDERSEN, C.; JONES, B. D. Comparative studies of policy agendas. Journal of European Public Policy, v. 13, n. 7, p. 959-974, 2006.

BAUMGARTNER, F. R.; JONES, B. D., editors. Policy dynamics. Chicago: University of Chicago Press, 2002.

BAUMGARTNER, F. R.; JONES, B. D. Agendas and instability in american politics. Chicago: University of Chicago Press, 1993.

BAUMGARTNER, F. R.; JONES, B. D. The politics of attention: how government priorizes problems. Chicago: University of Chicago Press, 2005.

Projeto CNPQ no 47414/2014-0. 
BAUMGARTNER, F. R.; JONES, B. D. The politics of information: problem definition and the course of public policy in America. Chicago: University of Chicago Press, 2015.

BAUMGARTNER, F. R.; JONES, B. D.; MORTENSEN, P. B. Punctuated equilibrium theory: explaining stability and change in public policymaking. In: SABATIER, P. A.; WEIBLE, C. M. Theories of the policy process. 3. ed. Boulder: Westview Press, 2014.

BAUMGARTNER, F. R.; JONES, B. D.; WILKERSON, J. D. Comparative studies of policy dynamics. Comparative Political Studies, v. 44, n. 8, p. 947-972, 2011.

BIRKLAND, T. A. An introduction to the policy process: theories, concepts, and models of public policy making. 2. ed. New York: ME Sharpe, 2005.

CAPElla, A. C. N.; SOARES, A. G.; BRASIL, F. G. Pesquisa em Políticas Públicas no Brasil: Um mapeamento da aplicação de modelos internacionais recentes na literatura nacional. In: ENCONTRO DA ABCP, 9., 2014, Rio de Janeiro. Anais Eletrônicos ... Rio de Janeiro: ABCP, 2014. Disponível em: $<$ http:// www.encontroabcp2014.cienciapolitica.org.br/resources/ anais/14/1403738847_ARQUIVO_ABCP_final.pdf. Acesso em 20 out. 2014.

COBB, R. W. et al. Agenda building as a comparative political process. American Political Science Review, v. 70, n. 1, p. 126-138, mar. 1976. http://dx.doi. org/10.2307/1960328.

COBB, R. W.; ELDER, C. D. The politics of agendabuilding: an alternative perspective for modern democratic theory. The Journal of Politics, v. 33, n. 4, p. 892-915, 1971.

COBB, R. W.; ELDER, C. D. Participation in American Politics: The dynamics of agenda building. Boston: Allyn and Bancon, 1972.

COHEN, B. C. The press and foreign policy. Princeton: Princeton University Press, 1963.

COMPARATIVE AGENDAS PROJECT - CAP. Disponível em: <http://beta.comparativeagendas. com/>. Acesso em: 9 out. 2015.

DOWNS, A. Up and down with ecology-the issue-attention cycle. The Public Interest, v. 28, p. 38, 1972.

FARAH, M. F. S. A contribuição da Administração Pública para a constituição do campo de estudos de políticas públicas. In: MARQUES, E.; FARIA, C. A. P. A Política Pública como Campo Multidisciplinar. São Paulo: Unesp; Rio de Janeiro: Fiocruz, 2013.

FARIA, C. A. P. Idéias, Conhecimento e Políticas Públicas: um inventário sucinto das principais vertentes analíticas recentes. Revista Brasileira de Ciencias Sociais, v. 18, n. 51, p. 21-29, 2003.

FESTINGER, L. A theory of cognitive dissonance. Evanston: Row, Peterson \& Co., 1957.
GREEN-PEDERSEN, C.; WILKERSON, J. How agendasetting attributes shape politics: Basic dilemmas, problem attention and health politics developments in Denmark and the US. Journal of European Public Policy, v. 13, n. 7, p. 1039-1052, 2006.

HOCHMAN, G.; ARRETCHE, M. T. S.; MARQUES, E. Políticas Públicas no Brasil. Rio de Janeiro: Fiocruz, 2007.

HUNT, V. F. The multiple and changing goals of immigration reform: a comparison of house and senate activity, 1947-1993. In: BAUMGARTNER, F. R.; JONES, B. D. (Ed.). Policy dynamics. Chicago: University of Chicago Press, 2002.

JOHN, P. Explaining policy change: the impact of the media, public opinion and political violence on urban budgets in England. Journal of European Public Policy, v. 13, n. 7, p. 1053-1068, 2006.

JOURNAL OF EUROPEAN PUBLIC POLICY. New York: Routledge, Taylor \& Francis Group. n. 7, 2006. Edição 13.

KATZ, E.; LAZARSFELD, P. Personal Influence. New York: The Free Press, 1995. Original de 1955.

KINGDON, J. Agendas, alternatives and public policies. 3. ed. New York: Harper Collins, 2003.

KLAPPER, J. T. The efects of mass communication. New York: Free Press, 1960.

LAZARSFELD, P. F.; BERELSON, B.; GAUDET, H. The people's choice: how the voter makes up his mind in a presidential campaign. 2. ed. New York: Columbia University Press, 1944.

LIPPMANN, W. Public opinion. New York: Harcourt Brace, 1922.

MARQUES, E.; FARIA, C. A. P. (Org.). A Politica Pública como Campo Multidisciplinar. São Paulo: Unesp; Rio de Janeiro: Fiocruz, 2013.

MCLEOD, M. C. The logic of positive feedback: telecommunications policy through the creation, maintenance, and destruction of a regulated monopoly. In: BAUMGARTNER, F. R.; JONES, B. D. (Ed.). Policy dynamics. Chicago: University of Chicago Press, 2002.

MCCOMBS, M.; SHAW, D. The agenda-setting function of mass media. Public Opinion Quarterly, v. 36, p. 176-195, 1972.

PRALLE, S. B. Timing and sequence in agenda-setting and policy change: a comparative study of lawn care pesticide politics in Canada and the US. Journal of European Public Policy, v. 13, n. 7, p. 987-1005, 2006.

PRINCEN, S.; RHINARD, M. Crashing and creeping: agenda-setting dynamics in the European Union. Journal of European Public Policy, v. 13, n. 7, p. 1119-1132, 2006

ROGERS, E. M.; DEARING, J. W. Agenda-setting research: Where has it been? Where is it going? Newbury Park: Sage, 1988. 
SCHATTSCHNEIDER, E. E. The semi-sovereign people. New York: Winston, 1960.

SOROKA, S. N. Agenda-setting dynamics in Canada. Vancouver, BC: UBC Press, 2002.

SOUZA, C. Políticas Públicas: uma revisão da literatura. Sociologias, ano 8, n. 16, p. 20-45, jul./dez., 2006.

TRAQUINA, N. O paradigma do agenda-setting: Redescoberta do poder do jornalismo. Revista ComunIcação $e$ Linguagens, v. 21-22, p. 299-318, 1995.
WOLF, M. Teorias da comunicação. Lisboa: Presença, 1986.

ZAHARIADIS, N. Ambiguity and multiple streams. In: SABATIER, P.; WEIBLE, C. M. (Eds). Theories of the policy process. 3. ed. Boulder: Westview Press, 2014.

Recebido: 9 out., 2015 Aceito: 27 nov., 2015 